\title{
Comparisons of CO and HI Dynamics in THINGS Galaxies
}

\author{
Bradley S. Frank and W. J. G. de Blok and the HERACLES Team \\ Astrophysics, Gravitation and Cosmology Centre \\ University of Cape Town \\ Rondebosch, 7700 \\ South Africa \\ email: bradley@ast.uct.ac.za
}

\begin{abstract}
We present recent results comparing the $\mathrm{CO}$ and $\mathrm{HI}$ dynamics of nearby galaxies that have been observed as part of the THINGS (HI) and the HERACLES (CO) surveys. We focus on 3 galaxies out of the 11 that have been analysed so far, and present velocity-fields and rotation curves of the $\mathrm{CO}$, which we compare with that of the HI.
\end{abstract}

Keywords. line: profiles, molecular data, galaxies: kinematics and dynamics

\section{Introduction}

Large HI surveys have mapped the neutral gas in local galaxies to low column densities with high spectral and spatial resolution. As such, studies of HI have been used to extract a variety of properties, such as star-formation rates, gas dynamics and, hence, the dark matter content in gas-rich galaxies. Molecular gas is the missing link between the neutral gas and stars, and the content and dynamics of molecular gas in nearby galaxies have been inferred through observations of the $\mathrm{CO}$ transitions, which is the most accesible tracer of $\mathrm{H}_{2}$. The majority of these observations, however, have lacked the sensitivity and area-coverage attainable with HI observations, prohibiting a single, consolidated study of the molecular gas dynamics of nearby galaxies and comparisons with HI.

Recent advances in high frequency radio receiver design has lead to more sensitive observations of $\mathrm{CO}$ in nearby galaxies, allowing for a mapping of the molecular gas content along the extent of the HI disk.

The aim of the current work is to provide a comparison between the gas dynamics of HI and $\mathrm{CO}$ in nearby galaxies, as observed with recent, high resolution surveys. The sample galaxies are part of the The HI Nearby Galaxy Survey (THINGS, Walter et al. 2008). In THINGS observations, the spectral resolution is $5 \mathrm{~km} \mathrm{~s}^{-1}$, and the spatial resolution $10^{\prime \prime} .34$ gas-rich galaxies of various morphologies were observed. Of these 34 galaxies, a dynamical analysis was performed on 19 (de Blok et al. 2008).

The HERA CO Line Extragalactic Survey (HERACLES, Leroy et al. 2009) is a survey of the molecular gas content of these galaxies, as measured by observing the $\mathrm{CO}(\mathrm{J}=2 \rightarrow 1)$ line. The spectral and spatial resolution of the HERACLES observations are $2.6 \mathrm{~km} \mathrm{~s}^{-1}$ and $13^{\prime \prime}$ respectively and is comparable to that of the THINGS survey, allowing for a comparison of the dynamics of both the neutral and molecular gas components.

Of the 34 galaxies observed in THINGS, 27 galaxies were observed in HERACLES. Of these, there are 15 galaxies which were analysed in the HI by de Blok 2008, of which 12 were detected in $\mathrm{CO}$. These are the galaxies on which we focus our present study.

We also briefly explore the interchangeability of $\mathrm{CO}$ and $\mathrm{HI}$ in the Tully-Fisher relation (TFR), as suggested in Lavezzi, T. E. 1998. 

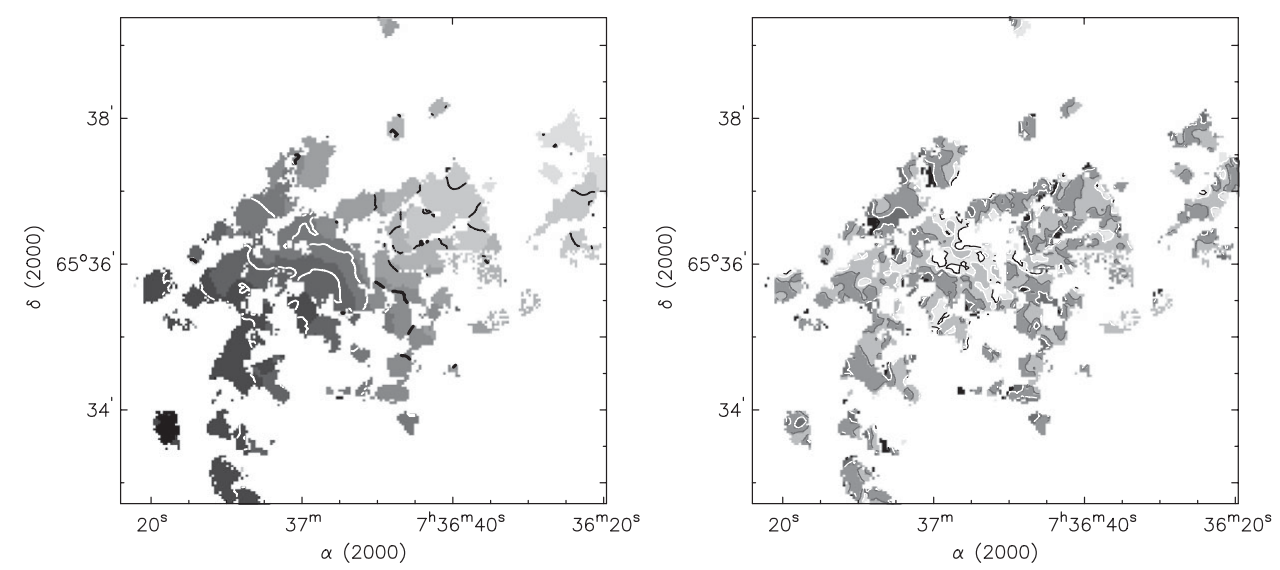

Figure 1. NGC $2403 \mathrm{CO}$ (left panel) and HI-CO (right panel) difference velocity-fields. In the $\mathrm{CO}$ velocity-field, levels are from $0 \mathrm{~km} \mathrm{~s}^{-1}$ to $225 \mathrm{~km} \mathrm{~s}^{-1}$ in steps of $25 \mathrm{~km} \mathrm{~s}^{-1}$, with $\mathrm{v}_{\mathrm{sys}}=132.8 \mathrm{~km} \mathrm{~s}^{-1}$. Difference velocity-field: Grayscale is plotted from $-10 \mathrm{~km} \mathrm{~s}^{-1}$ to $10 \mathrm{~km} \mathrm{~s}^{-1}$ in steps of $5 \mathrm{~km} \mathrm{~s}^{-1}$. Black contours correspond to an absolute difference of $10 \mathrm{~km} \mathrm{~s}^{-1}$, white to $5 \mathrm{~km} \mathrm{~s}^{-1}$ and dark grey to $0 \mathrm{~km} \mathrm{~s}^{-1}$.

\section{Methods and Techniques}

We derive a peak velocity-field by fitting Gauss-Hermite polynomials of order 3 to each pixel in a $\mathrm{CO}$ data cube, in accordance with the method prescribed in de Blok et al. 2008.

We also derive the difference velocity-field by subtracting the CO velocity-field from the HI velocity-field derived in de Blok et al. 2008.

We compute two rotation curves from the $\mathrm{CO}$ velocity-field using a tilted ring model with the GIPSY program ROTCUR for each galaxy. The first is computed by simply applying the geometrical parameters as derived from the THINGS data (the THINGS-Model) and as presented in de Blok et al. 2008; the second is computed using an independent ROTCUR analysis where we solve for all the dynamical parameters, in addition to the circular velocity (the All-Free case). We assume only circular rotation.

\section{Results for NGC 925, 2403 and 5055}

We present a summary of results from a representative subsample of galaxies (NGC 2403, NGC 5055 and NGC 925).

$N G C 2403$ is a late-type gas rich spiral. The velocity and difference velocity-fields are plotted in Figure 1. The difference velocity-field indicate that the differences between the $\mathrm{CO}$ and $\mathrm{HI}$ are small and localized. This is to be expected, since the $\mathrm{CO}$ emission is likely to be associated with regions of turbulence and star-formation. Figure 2 shows that the CO THINGS-Model rotation curve agrees quite closely with that of the HI, out to a radius of about 150 arcseconds. The $\mathrm{CO}$ All-Free rotation curve shows a similar trend, and the departure between the $\mathrm{HI}$ and $\mathrm{CO}$ rotation curves correlates with a low filling-factor of the tilted rings.

$N G C 5055$ is a late type LINER with flocculent spiral arms evident in the HI and in the $\mathrm{CO}$. The velocity and difference-velocity-fields are plotted in Figure 3. The difference-velocityfield shows that there is a good agreement between the $\mathrm{HI}$ and the $\mathrm{CO}$ velocities. Part of the differences in the inner parts of the galaxy can be attributed to a rapidly rotating molecular circum-nuclear disk.

There is a good agreement between the HI and the CO-All-Free rotation curves from 30 arcseconds outwards to the extent of the $\mathrm{CO}$ emission.

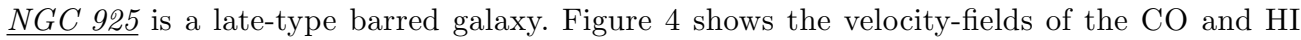
respectively. A comparison with the SINGS (Kennicutt et al. 2003) image and Figure 4 shows that the $\mathrm{CO}$ emission is limited to a region along the bar on the approaching side of the galaxy 

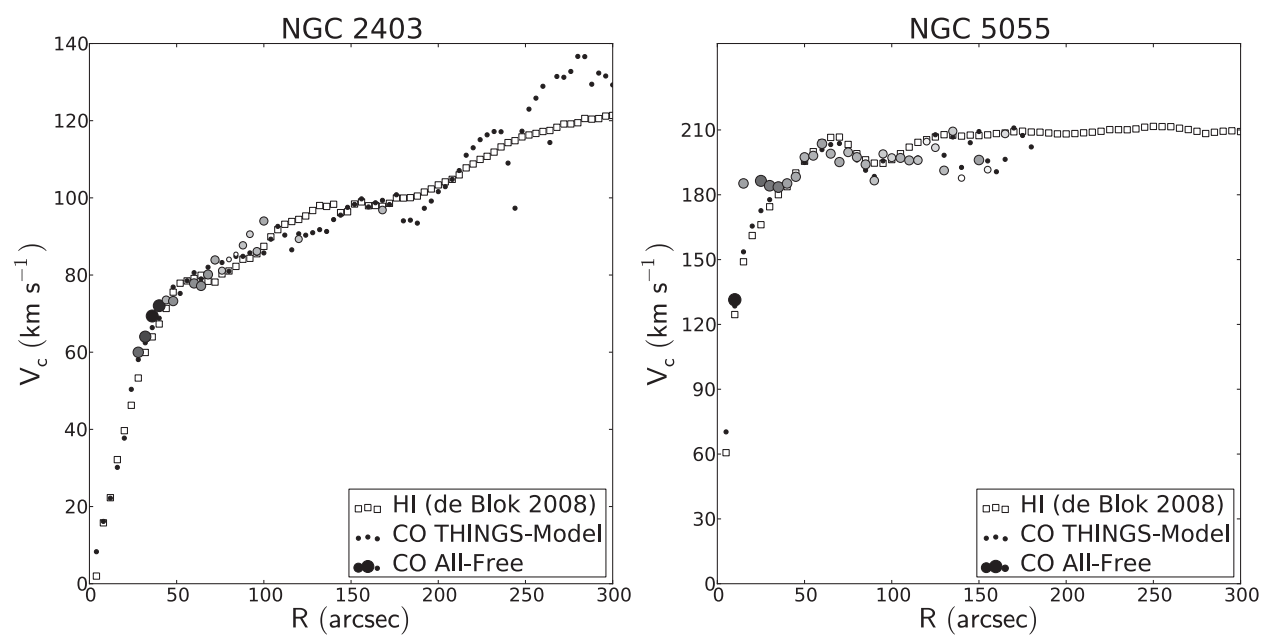

Figure 2. NGC 2403 and NGC 5055 CO rotation curves. The size and color of the CO All-Free points scale with the filling factor. Here we plot points with a filling factor greater than $5 \%$.
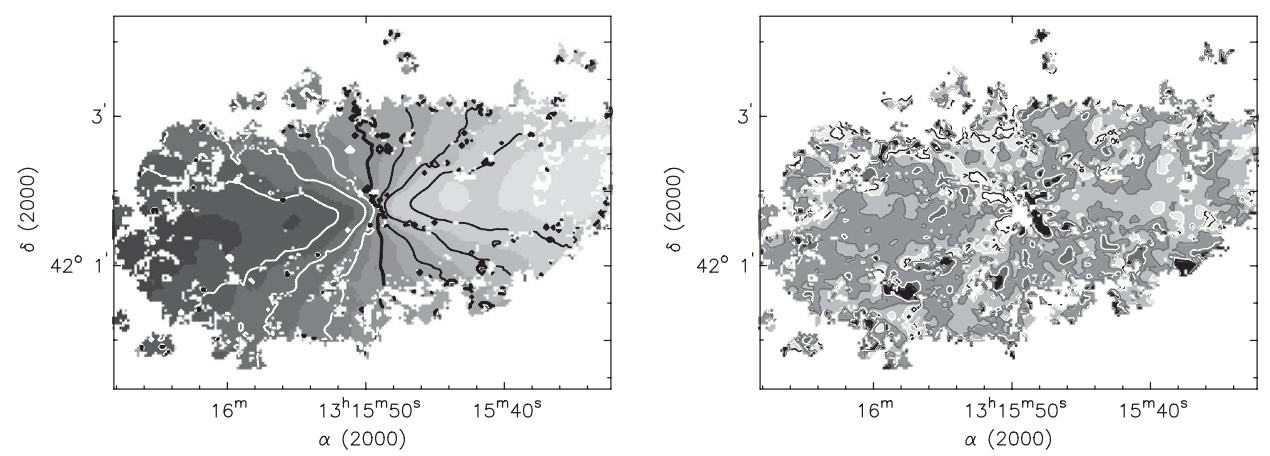

Figure 3. NGC $5055 \mathrm{CO}$ velocity-fields, as in Figure 1. In the CO velocity-field, levels are from $225 \mathrm{~km} \mathrm{~s}^{-1}$ to $725 \mathrm{~km} \mathrm{~s}^{-1}$ in steps of $50 \mathrm{~km} \mathrm{~s}^{-1}$, with $\mathrm{v}_{\mathrm{sys}}=496.8 \mathrm{~km} \mathrm{~s}^{-1}$. Contours in the difference field are as in Figure 1.

presenting an additional challenge in rotation curve derivation. The low $\mathrm{CO}$ intensity and the concentration of $\mathrm{CO}$ along the bar means that it is not straightforward to derive a rotation curve that is an accurate represention of the rotational velocity.

\section{Conclusion}

There is a good agreement between the dynamics of the HI and the CO for the galaxies presented. The THINGS-Model parameters are a suitable first estimate for the CO dynamics, but independent rotational parameters should be derived from the CO data. Local differences can be attributed to regions of turbulence in the ISM, as well as the additional motions due to a possible circum-nuclear disk in NGC 5055 and the bar in NGC 925.

The HI and CO global profiles are plotted in Figure 5. We see that substantial differences in the velocity widths between both components are possible, which implies that a CO TFR needs to be calibrated independently of an HI TFR. This is important when considering the observations of high-redshift $\mathrm{CO}$ as a proxy for $\mathrm{HI}$ in a general TFR.

In future work we will present rotation curves of all the galaxies analyzed in this study (Frank et al., in prep.), where we compare the dynamics of the $\mathrm{CO}$ and the HI, in addition to constructing new mass-models including the molecular gas. This work aims to provide a 

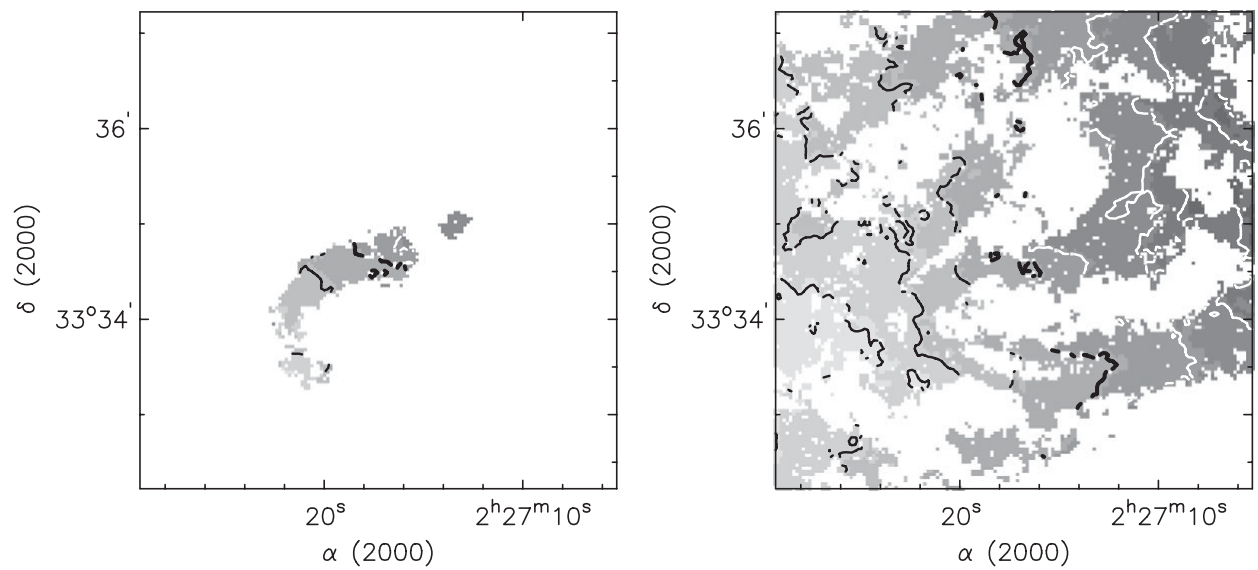

Figure 4. NGC $925 \mathrm{CO}$ (left panel) and HI (right panel) velocity-fields. Levels are from $425 \mathrm{~km} \mathrm{~s}^{-1}$ to $700 \mathrm{~km} \mathrm{~s}^{-1}$ in steps of $25 \mathrm{~km} \mathrm{~s}^{-1}$, with $\mathrm{v}_{\mathrm{sys}}=546.3 \mathrm{~km} \mathrm{~s}^{-1}$.
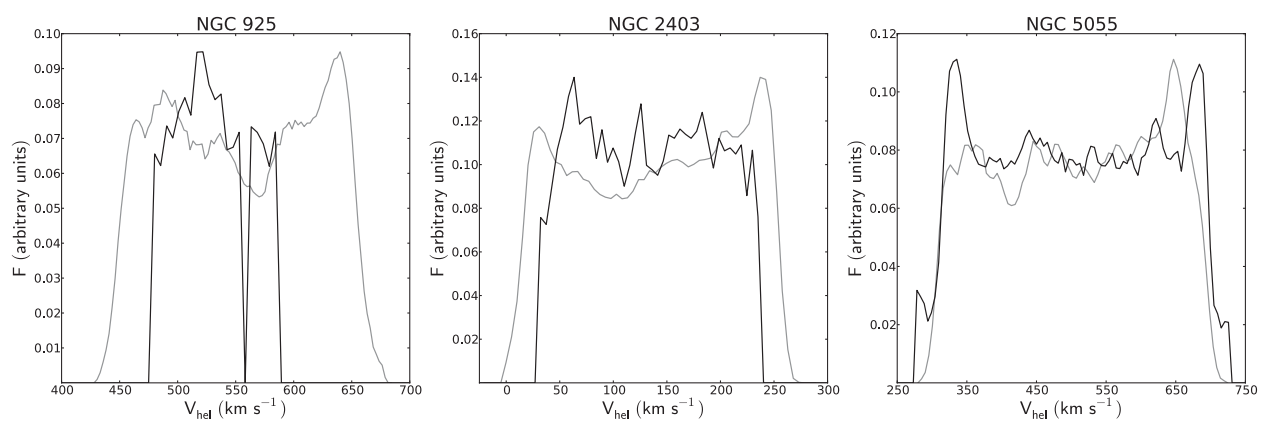

Figure 5. HI (grey) and CO (black) global profiles for NGC 925, NGC 2403 and NGC 5055.

template for future studies of HI and CO with next-generation instruments (e.g. the SKA and ALMA), since we can associate differences in the morphologies of global profiles of HI in CO in nearby galaxies with an in-depth comparison of the dynamics.

\section{References}

Walter F., et al. 2008, AJ, 136, 2563

de Blok, W. J. G. et al. 2009, AJ, 136, 2648

Leroy Adam, K. et al. 2009, AJ 137, 4670

Kennicutt, R. C., Jr. et al. 2003, PASP, 115, 928

Lavezzi, T. E. \& Dickey, J. M. 1998, AJ 116, 2672 